\title{
STATUS OF THE FERMILAB TEVATRON PROJECT
}

\author{
A.V.Tollestrup* \\ Fermi National Accelerator Laboratory ${ }^{\dagger}$ \\ Batavia, Illinois
}

\begin{abstract}
SUMMARY
A brief survey of the Fermilab Tevatron is presented, with emphasis on the magnet development. Some operational aspects of strings of up to 24 magnets are presented.
\end{abstract}

\begin{abstract}
$\therefore$ A great many individuals under the direction of R.R. Wilson have contributed enormously to this project. Recognition of some of these contributions is given by teans of references -- others are presucably having their deeds recorded by Abou Ben Adhem and will be rewarced at an appropriate time and place.
\end{abstract}

\footnotetext{
- Operated by Universities Research Association, Inc., under contract with the L.S.Department of Energy.
} 
The Energy Doubler is a proton accelerator that receives its injected beam from the Fermilab Main Ring at $150 \mathrm{GeV}$ and is capable of accelerating the beam to $1000 \mathrm{GeV}$. Fixed target physics as well as colliding beam experiments are planned and both slow (few seconds) and fast extraction $(\sim 1 \mathrm{msec})$ are planned at intensities of $2 \times 10^{3}:$ protons per pulse. ${ }^{1}$

In the ring there are 760 dipoles and 240 quadrupoles. These elements, consisting of four dipoles and one quadrupole per half cell, are all connected in series and operate at a current of about $4500 \mathrm{~A}$. The field in the dipoles is near $4.5 \mathrm{~T}$ and the quads have a gradient of about $20 \mathrm{kG} /$ inch.

The magnets are powered by six $2 \mathrm{kV}$ supplies distributed around the ring that are capable of generating a $75 \mathrm{GeV} / \mathrm{sec}$ rasp rate. ${ }^{2}$ At flat top a single very well regulated low voltage, low ripple, holding supply will maintain the current during extraction or colliding beam experiments.

Since at full field each magnet stores almost $0.5 \mathrm{MJ}$, it is obvious that a sophisticated protection system is necessary to protect the magnets during a quench of any one of them. The system planned, 2 uses a micro-processor to monitor the voltage across each half cell and compares it with all the others. In the event of a quench the increasing resistive drop is detected and identified as a quench condition. That half-cell is then shorted and internal heaters fired to drive the four magnets normal as quickly as possible. The energy is absorbed in the cold part of the magnet structure, which increases its temperature to less than $150^{\circ} \mathrm{K}$ and the energy in the rest of the ring is dumped into resistors.

The refrigeration system ${ }^{3}$ consists of a very large Central Helium 
Liquefier Plant and 24 satellite stations placed around the ring. The Central plant is capable of producing up to $5000 \mathrm{l} / \mathrm{hr}$ of liquid helium. In normal operation each satellite is supplied with $92 \ell / h r$ and acts as an amplifier to produce 966 watts of cooling at $4.2^{\circ} \mathrm{K}$. Each satellite feeds a string of about 32 dipoles and 8 quads. The satellites in a stand-alone wode are capable of maintaining the ring superconducting in case of failure of the Central Plant.

\section{PRESENT STATUS}

The Tevatron will be the first large high energy accelerator employing superconducting technology. Not only is it planned as an accelerator, but also it will be used as a storage ring. This is an exceedingly ambitious project with no antecedents for guidance and consequently we have concentrated our efforts on answering as many of the new and fundamental problems as possible. So far the following five areas have mainly occupied our attention:

1. Methods to fabricate magnets

2. Measurement of the magnet characteristics

3. Behavior of strings of magnets both from a cryogenic point as well as protection under quench conditions

4. Effect of high energy protons striking the superconducting coils

5. Development of the necessary cryogenic plant.

We address some of these areas here.

\section{Magnet Fabrication}

The magnet ${ }^{4}$ consists of two shells (see Figure 1). The inner subtends a half angle of $\sim 72^{\circ}$ and the outer $\sim 36^{\circ}$. The wire is trapezoidal with dimensions of . 307" wide, inside height .045" and outside 


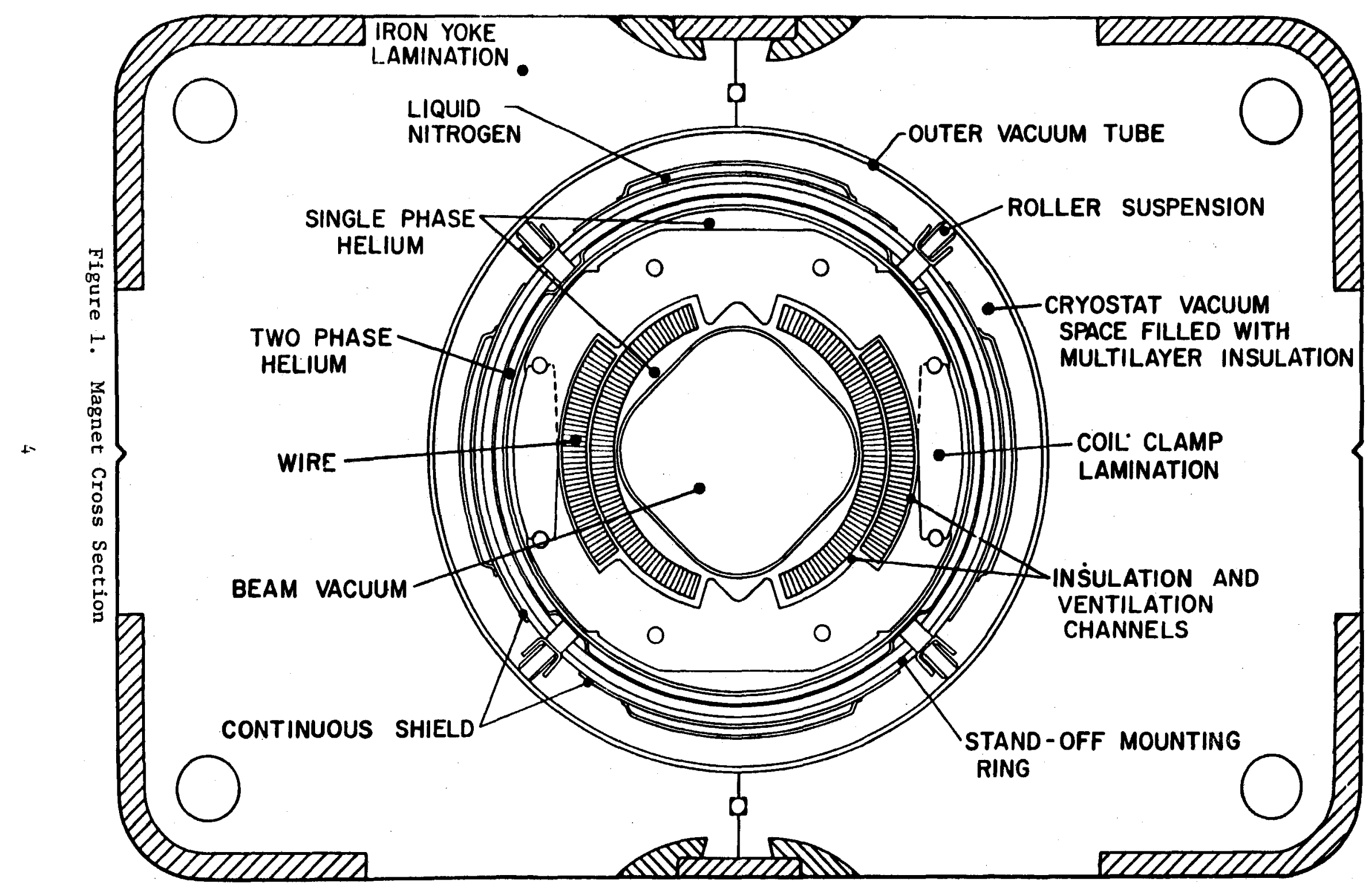


height .056". It will carry $>5300 \mathrm{~A}$ of $5 \mathrm{~T}$, and is insulated by a double overlap wrap of 1 mil Kapton and a spiral wrap of B-staged glass tape.

The inner half-coil of 3.5 turns is wound flat and pressed into shape in a 3000 ton, 24' long precision press. The cross section of the mold is controlled by constructing it from precision stamped laminations.

The outer half-coil of 21 turns is wound over the inner one, using it as a mandrel. This whole structure is again placed in a precision mold, pressed into shape with a second 3000 ton press and cured to its final shape at about $240^{\circ} \mathrm{F}$ (see Figure 2).

After the half coils are molded, they are measured for circumferential length with a gauge that can apply pressure up to $2000 \mathrm{lb} / \mathrm{linear}$ inch on either the inner or outer coil. The size at $1500 \mathrm{Ib} /$ linear inch is measured at 10 places along the axis and on each side of the coils. The average of these 10 readings can be controlled to about \pm 2 mils. Magnet 1172 had measurements as given below:

\section{Magnet 172 Coil Sizes}

Inner

Outer

$\underline{\text { Right }} \quad \underline{\text { Left }} \quad \underline{\text { Right }}$

$\begin{array}{lllll}\text { Top } & 2.117 \pm .002 & 2.117 \pm .002 & 1.272 \pm .002 & 1.272 \pm .002 \\ \text { Sottom } & 2.115 \pm .0015 & 2.116 \pm .002 & 1.273 \pm .003 & 1.2725 \pm .002\end{array}$

The error is the rms fluctuation of the 10 measurements. It is very important to control the size of the coil halves very accurately or when the coil is collared the median plane will not be centered and a quadrupole moment will arise. For instance, a shift of the median plane of $.001 "$ of the two coils relative to the fixed corners in the collars will generate a skew quadrupole, $a_{1}$, of $2 \times 10^{-4}$ inches $^{-1}$. 

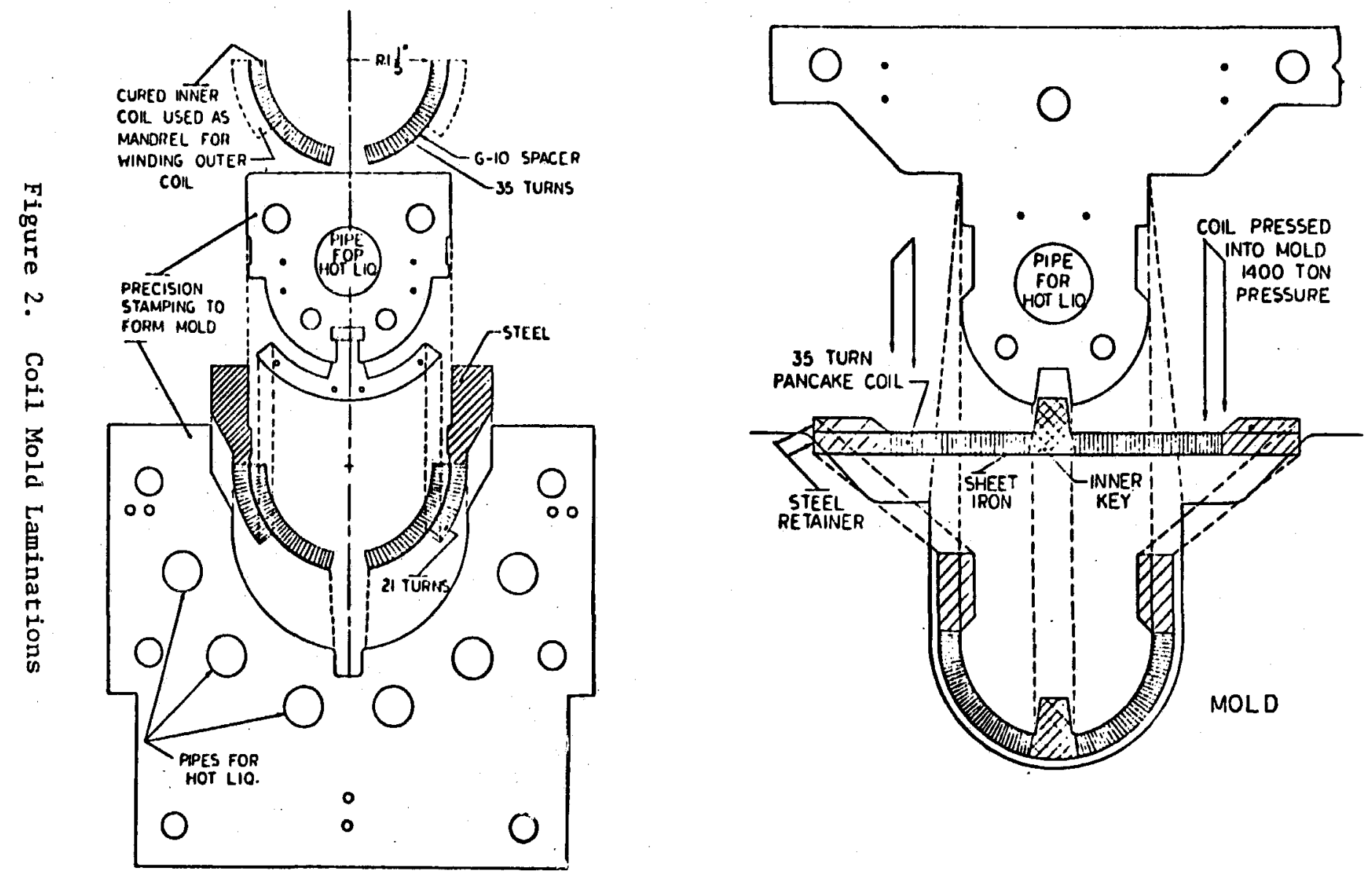
A second reason that the coils must be accurately sized is that the "preload" on the coils when collared must be carefully controlled. In order that the coils not move away from the key surfaces in the collar, it is necessary to compress the inner coils with an elastic force of $1300 \mathrm{lb} /$ inch and the outers with $500 \mathrm{lb} /$ inch. However, when the coils are cooled, they shrink more than the collars. The stressstrain curve for the coils, both warm and cold, has been studied ${ }^{5}$ and it is found that an initial room temperature compression of the outer coils of $1500 \mathrm{lb} /$ inch and the inners by $4000 \mathrm{lb} /$ inch is necessary in order to achieve the required preload when the coil is cold. These compressions give a differential Young's modulus for the coils of about $10^{6}(\mathrm{psi})^{-1}$.

The control of coil size is crucial to understand, and additional techniques are still being developed to inprove and simplify the accurate fabrication of coils.

After the magnet is collared, the field in the horizontal plane is measured at room temperature. ${ }^{6}$ This is done by inserting into the magnet bore a set of 12 long loops $0.195^{\prime \prime}$ wide $\times 24^{\prime}$ long, spaced $.215^{\prime \prime}$ to The coils are supported in a stainless steel tray and cast into epoxy for stability. The magnet is then excited at $11 \mathrm{~Hz}$ with a current of about $10 \mathrm{~A}$ and a lock-in amplifier used to measure, the difference of the induced voltages between adjacent loops. This gives a number proportional to the gradient of the field. The coil zust be calibrated since differences in area are much greater than the change in $B$ over the aperture $\left(\delta B / B \sim 10^{-4}\right)$. We have been able to refine these measurements sufficiently so that we can determine $b_{1}, b_{2}$, $b_{3}$, b 4. These parameters as measured at room temperature without an iron yoke can be related to their final value with a yoke and with the 
coil superconducting.

The block diagram of the factory then can be outlined as in Figure 3.

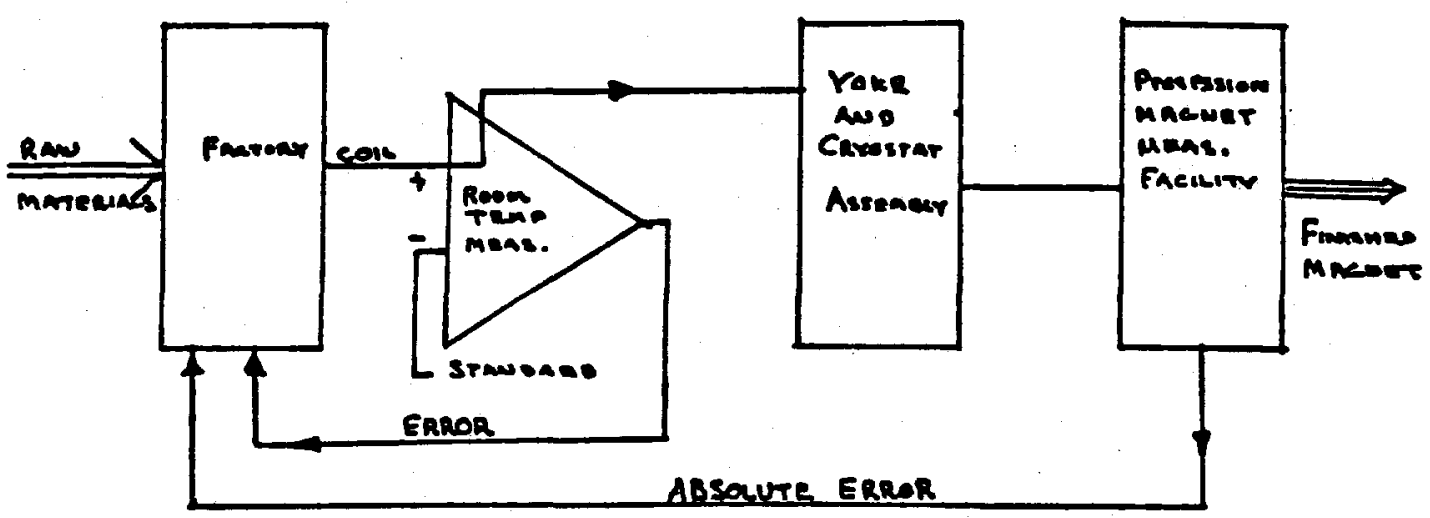

Figure 3. Block Diagram of Factory

The room temperature measurements provide a means for immediately gonitoring the quality of the collared coil.

If deviations outside of acceptable limits occur in a coil, it can be set aside for recollaring, and appropriate adjustments made in the next coil to correct the trouble. The long-term stability of the system is controlled by a slow feed-back loop provided by the precision magnet test facility.

Two very important points are implicit in the operation of this scheme. First, the factory must make reproducible coils. Fluctuations from coil to coil cannot be removed by the fast feed-back loop. The reproducibility is controlled by the very precise tooling used in the manufacture. It was necessary to invent the "laminated tooling" in order to achieve the necessary small tolerances."

The second important discovery came when it was realized that absolute dimensional accuracy is not required anymore. Consider a 
coil of the shape shown below (Figure 4).

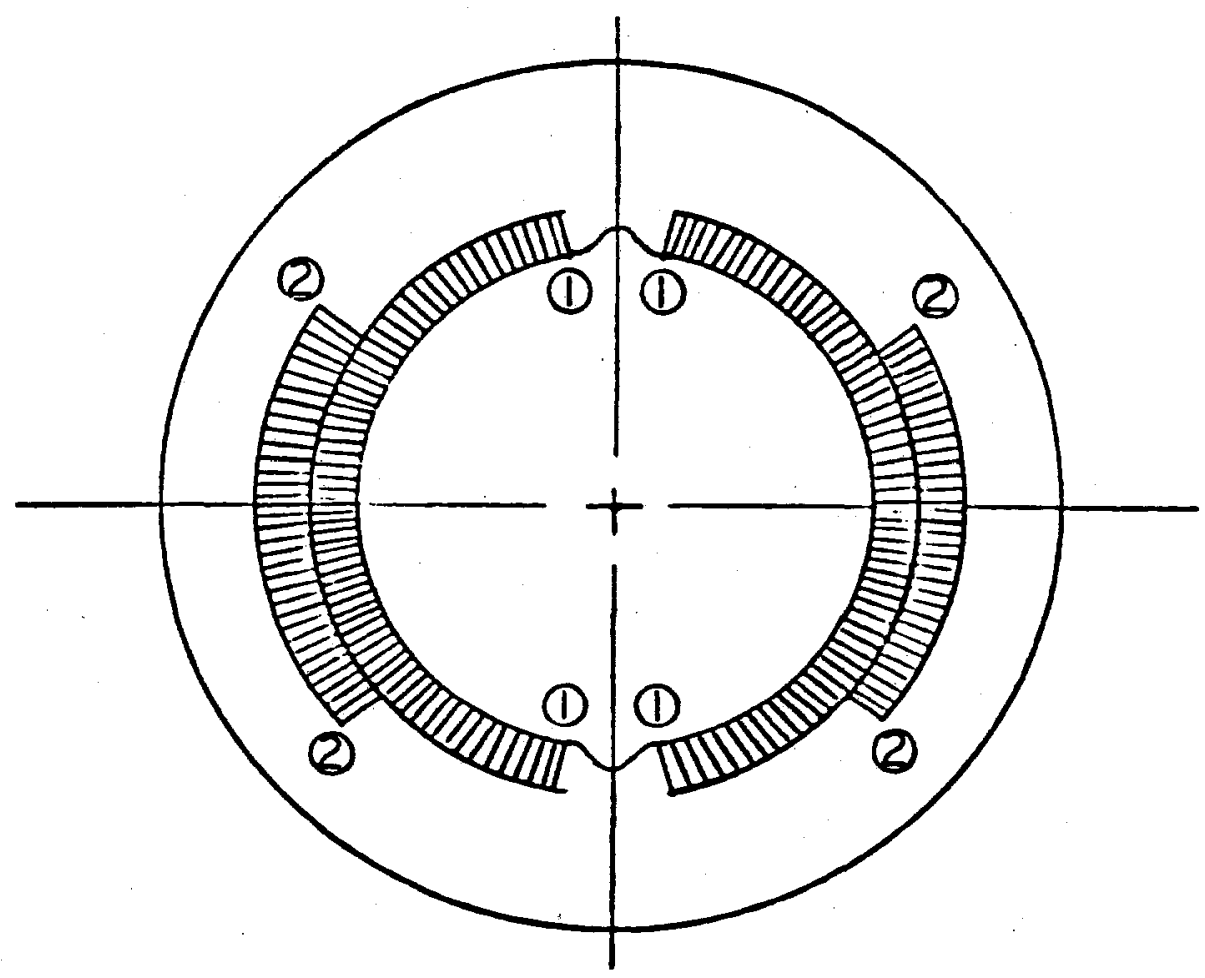

Figure 4. Coil Cross Section

The structure is designed in such a way that the stainless steel shim stock can be inserted before collaring, at the points labeled (1) and (2). Thus the angles of the coil blocks can be changed by small amounts at will.

Consider the harmonic structure of such a coil. The calculated zovements in $\delta B / B$ at $l^{\prime \prime}$ are given below (integrated through the cagnet).

$$
\begin{aligned}
& b_{2}=0 \\
& b_{4}=1.1 \times 10^{-4} \\
& b_{6}=4.4 \times 10^{-4} \\
& b_{3}=-12.1 \times 10^{-4} \\
& b_{10}=3.6 \times 10^{-4} \\
& b_{12}=-0.8 \times 10^{-4} \\
& b_{14}=.07 \times 10^{-4}
\end{aligned}
$$

These coefficients give a $B(x) / B_{0}$ (integrated through magnet) as a 
function of $x$ as shown in Figure 5.

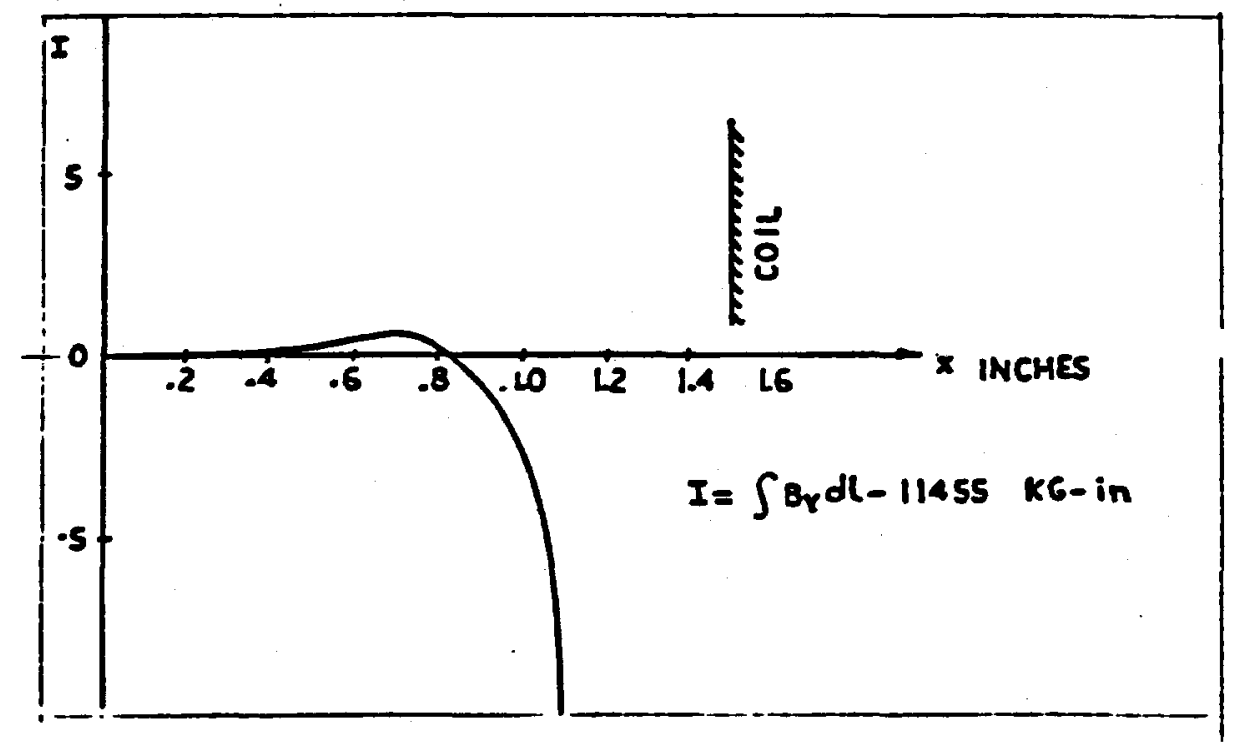

Figure 5. Variation of the Integral of B Through the Nagnet as a Function of Radius

With a little work it can be shown that for small changes of the coil shape only the multipoles below the 14-pole will change. Thus, if the coil is not exactly circular, but perhaps a little elliptical or square, or some other shape, the higher multipoles which are detergined by the Fourier components of the current block corners will be nearly the same as in the correct coil shape. However, the decapole terms and below will be different and there are eight places shims can be inserted into the coil structure to modify these components.

Thus we arrive at an important point. If the factory is reproducible, a coil can be built, measured, and the next one corrected to have a uniform field without having to produce a coil whose absolute dimensions are known. This process will leave a small random fluctuation in the central field, Bo, but this is very small and the orbit distortions can be corrected by the horizontal correction elements 
that are necessary in any event.

We now present some data gathered over the last two years. Over 170 coil assemblies have been constructed during the development of the coil fabrication technique. Some of these coils are suitable for accelerator tests after their insertion into a cryostat. Some are being used in rather extensive systems tests to be described later, and many of the rest will be useful for beam tramsport. To get some idea of the overall quality of these magnets, for use in beam transport systems, it may be said that the random errors have always been small enough so that a single pass through 740 such randomized magnets leaves the phase space inside $\pm \frac{1}{2} "$ essentially an undisturbed ellipse.

The last major hurdle was to close the room temperature feedback loop, which was done in January 1979. In the meantime the magnets have been shortened by one foot in order to accomodate shielding in the quadrupole cryostats which will help protect the dipole coils from stray beam at injection and extraction. Since April 1979. the new series of 2I' magnets has been underway. On the basis of what we have learned it is expected that "Accelerator quality" magnets will be produced. The factory has been operated at a level of five magnets per week for short test periods and should be capable of 10/week. The main problems expected are in the quality control area.

\section{MAGNET MEASUREMENTS}

We begin by presenting data obtained in vertical dewar tests of the collared coils. ${ }^{7}$ The yoke and cryostat are not yet assembled and only measurements of training, peak field, ramp rate dependence, change in radius of the coil, azimuthal motion of the wire, transfer constant, and residual field due to persistent currents are measured. 
Figure 6 shows the training history of one of the recent coils, $\$ 167$, and Figure 7 gives the $B$ dependence of the quench point. A typical load line and maxisum quench point are given in Figure 8 . With the iron, the peak field point is $1.1 \mathrm{~B}_{0}$ at the key of the inner coil, and without iron the high field point is at the inner turn at the very end of the coil. The end turns are spaced out slightly to reduce the field at the end.

Now to examine the statistics of a number of coils we show the following three histograms. Figure 9 shows the number of quenches to reach $95 \%$ of $I_{\max }$, and Figure 10 shows the percent of expected short sample current that the coil achieves. The short sample curve of each reel of wire is measured after being received from the manufacturer. Finally, Figure 11 shows the distribution of peak quench currents obtained.

The ramp rate dependence of the coils is determined by both the heat generated during ramping as well as by the cooling. The coils initially were wound with Stay-Brite-coated wire. Subsequently it has been determined that eddy current losses in the coil package were too high with such good contact between the strands of wire. Consequently we are now using Ebonol-(copper oxide) coated strands and the losses are essentially all due to hysteresis.

COIL LOSSES

\section{Ebonol Stay-Brite}

Hysteresis $200 \mathrm{~J} \quad 200 \mathrm{~J}$

Eddy Current $\sim 20 \mathrm{~J} \quad 160-560 \mathrm{~J}$

The above numbers are for the following ramp: 


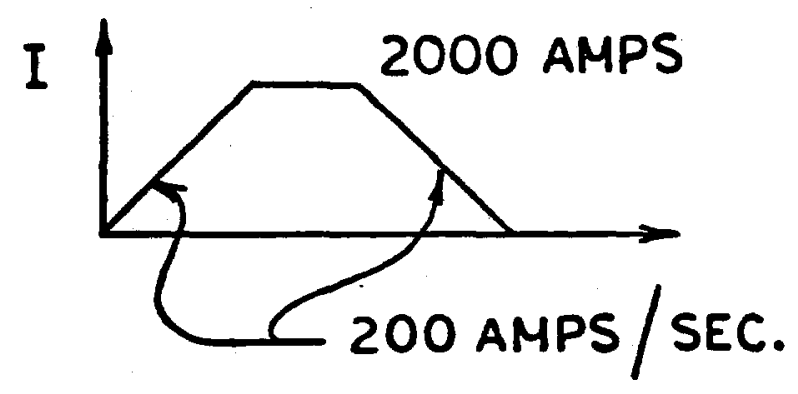

The collars must contain a force in the $x$ direction of $6000 \mathrm{lb} /$ inch, which tends to blow the coil apart. Fatigue studies were made of the collaring system and a lifetime in excess of $10^{8}$ cycles is now expected. Figure 12 shows the radial deflection as a function of coil current. It is accurately parabolic and a histogram of the deflections of 23 magnets is given in Figure 13. There are other elastic motions of the wire. These have been studied and their net effect on the field shape is well within the level of the correction circuits. It is worth noting that the persistent currents generate a rather strong sextupole field ( $\sim 10$ gauss) which must be corrected and in general, in superconducting magnets, there must always be dynamic correction elements which are under program control and with a magnitude of a few $\times 10^{-}$. After the magnet coil is assembled in its cryostat ${ }^{B}$ it is given a complete set of precision magnetic tests in the Magnet Test Facility.9 This Facility consists of six test stands where a magnet may be connected to power and refrigeration, and a 1500 watt refrigerator capable of cooling the system. Each magnet is vacuum tested, tested cryogenically, trained, its ramp rate dependence measured as well as the energy loss per cycle at various B's. Finally, a complete set of dc and ramped field harmonics is measured at eleven different currents. The $\int B \cdot d l$ is measured by a combination of NMR and Hall probes that are Eoved through the magnet automatically by the computer. This complete 


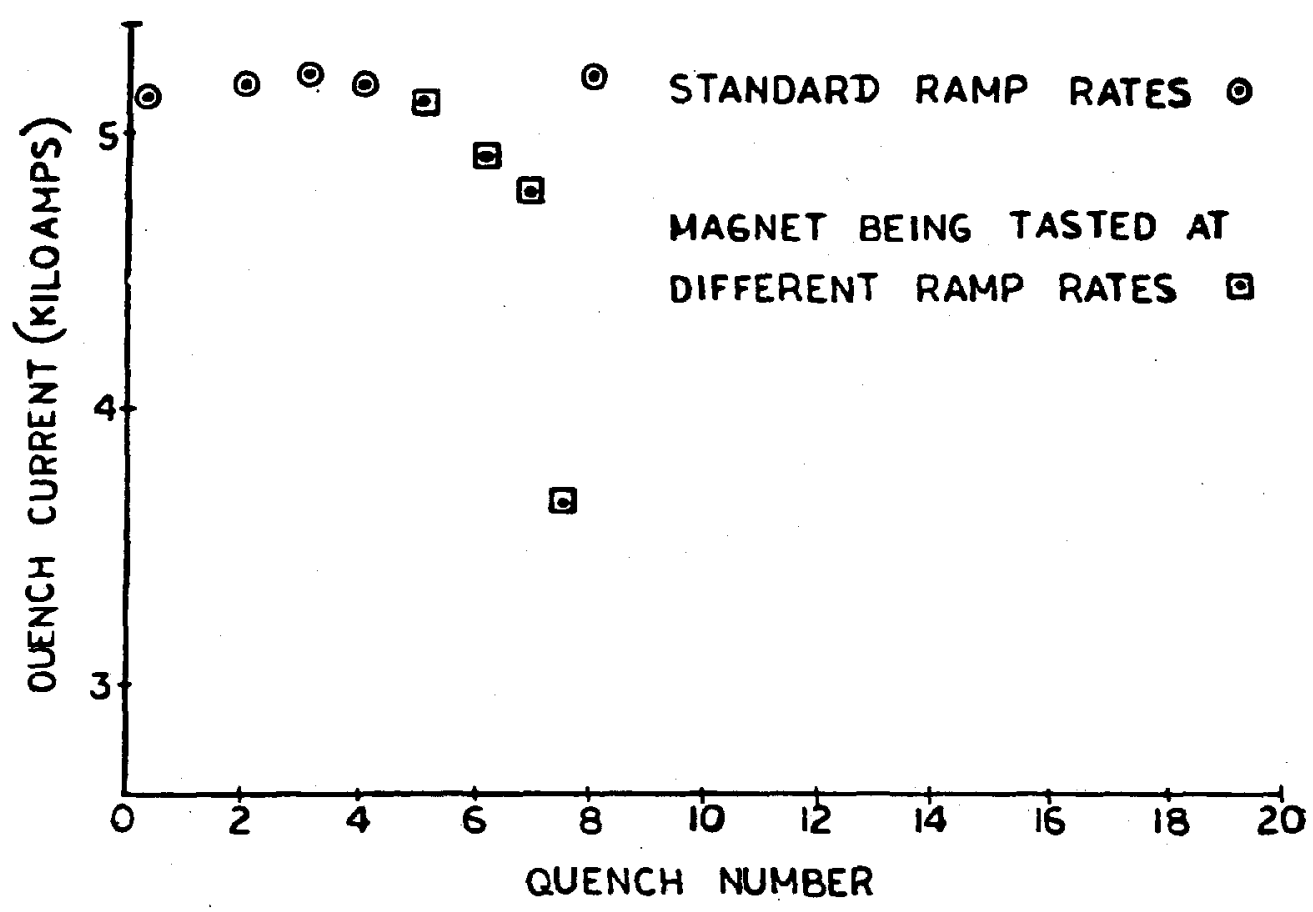

Figure 6. Training - 167

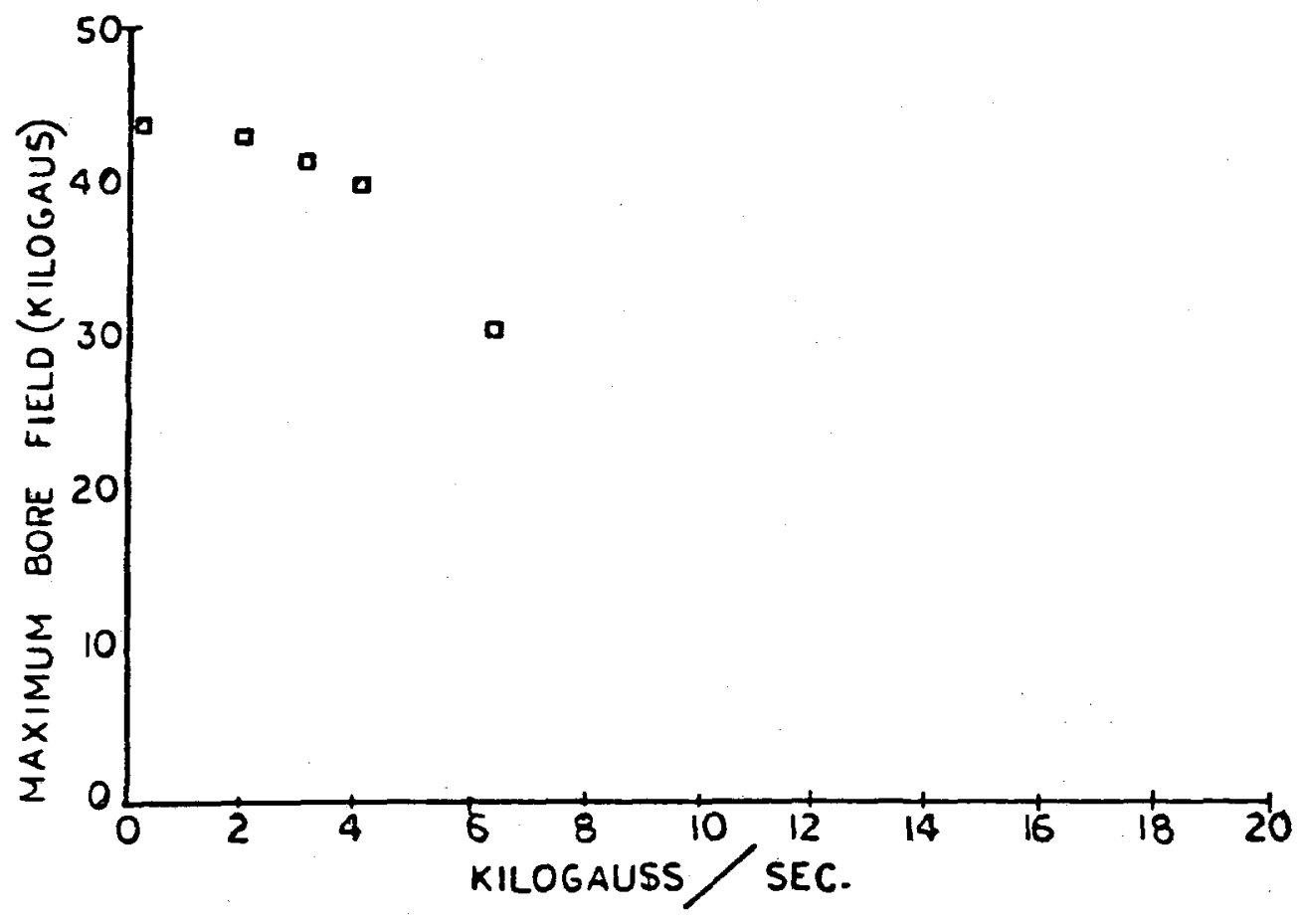

Figure 7. B Curve 


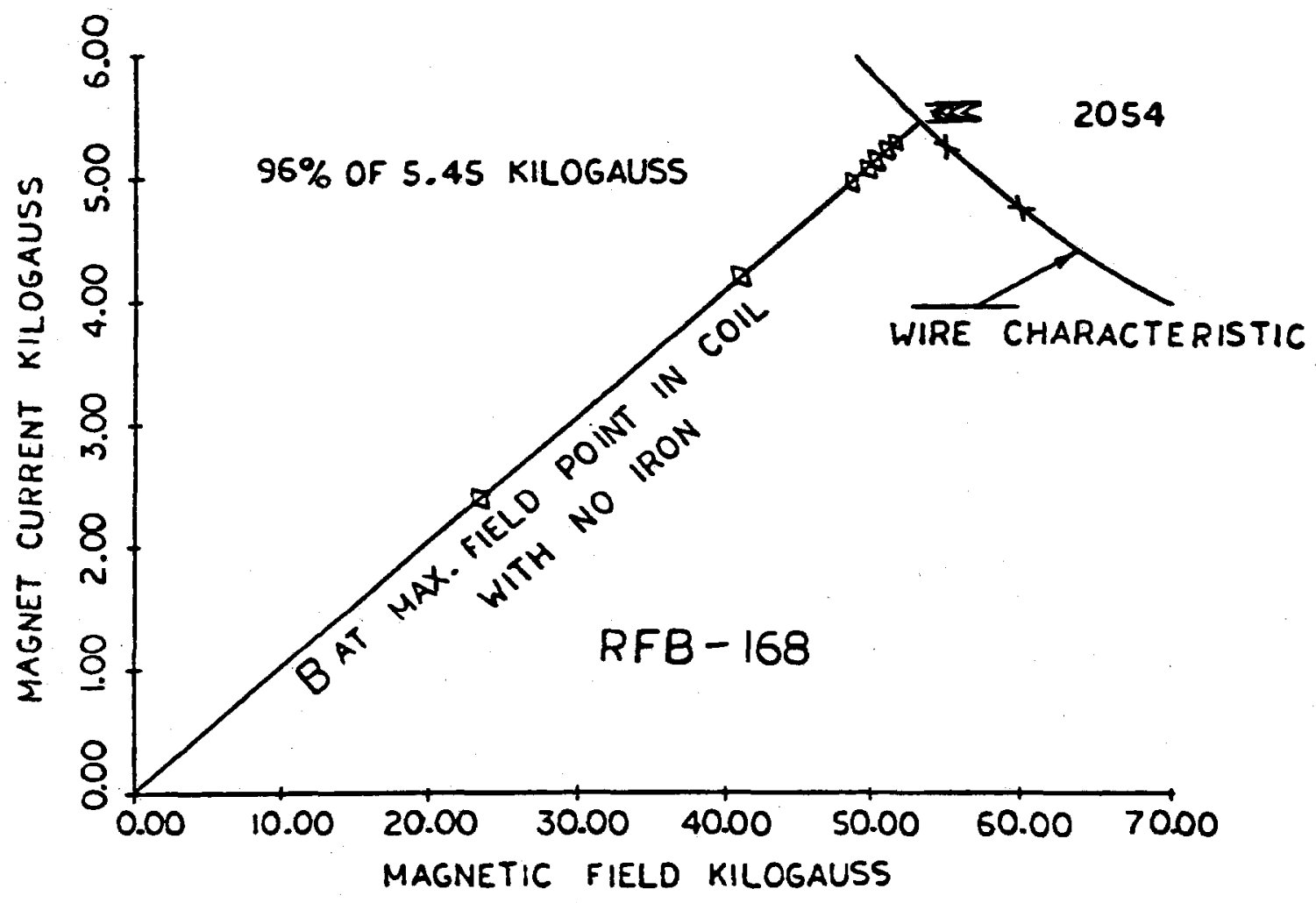

Figure 8. Load Line and Short Sample Limit

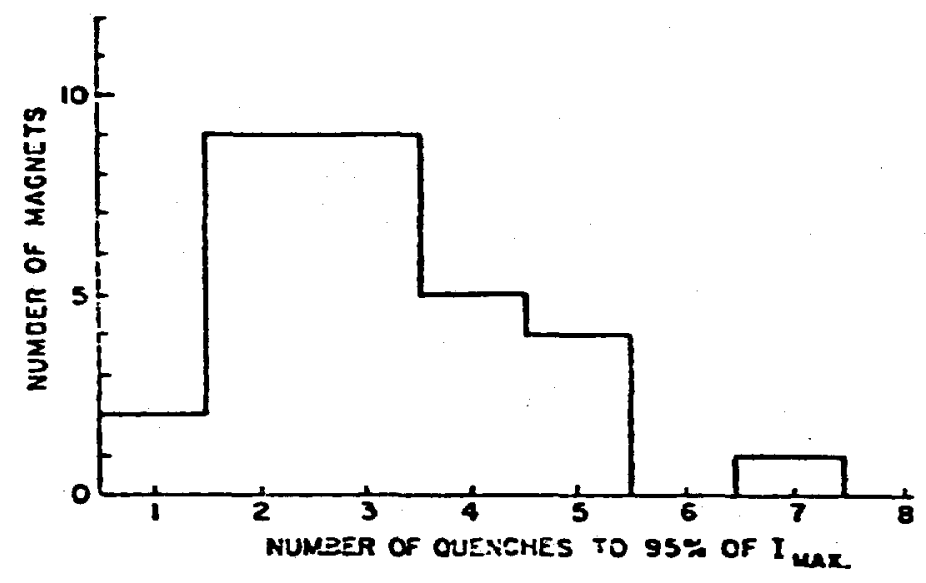

Figure 9. Train Current Distribution 


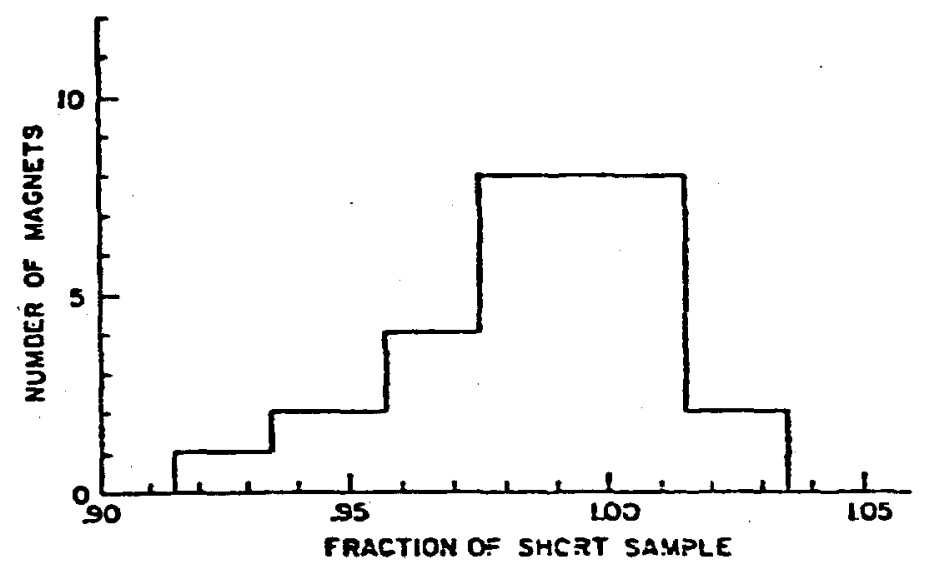

Figure 10. Distribution of Final Training Current as a Fraction of Short Sample Limit

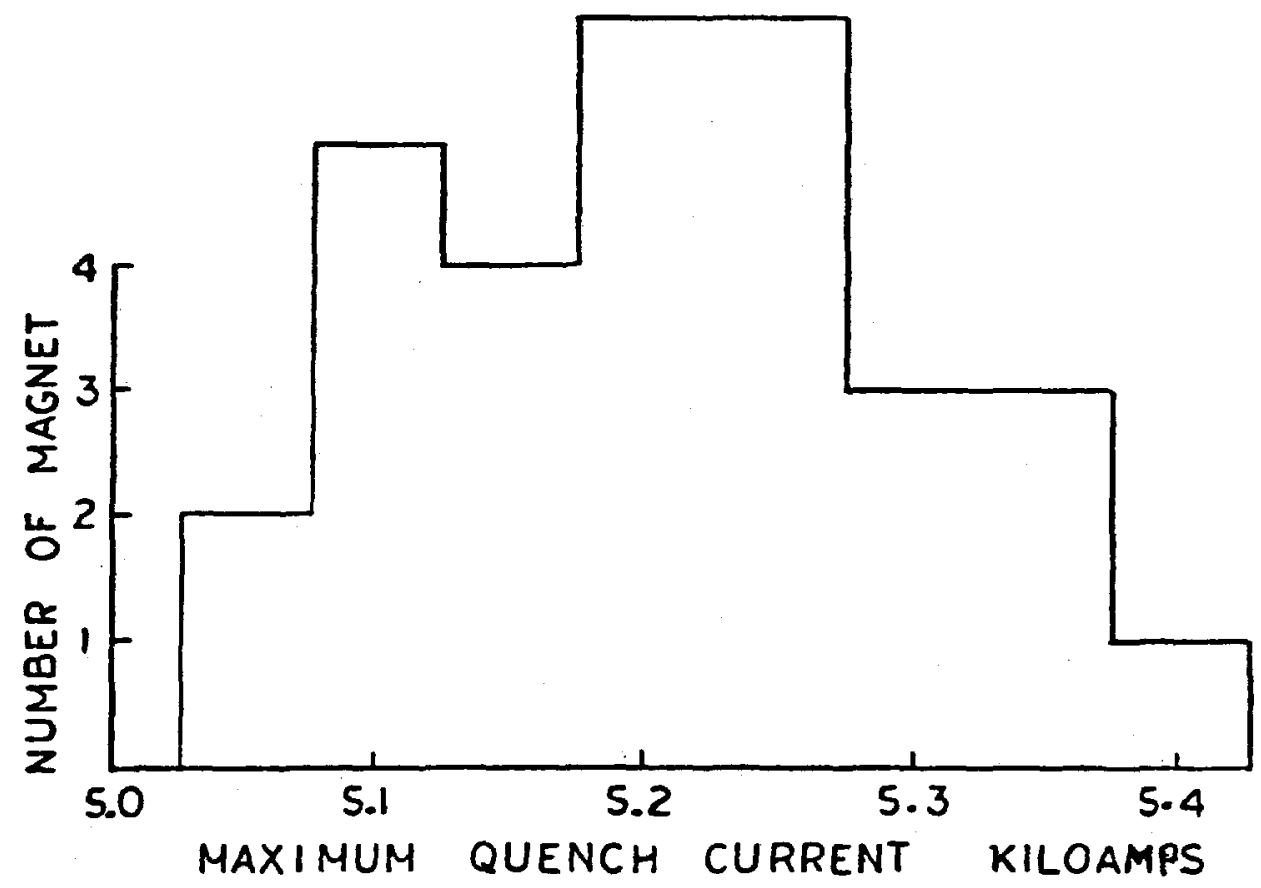

Figure 11. Distribution of Max. Quench Current 


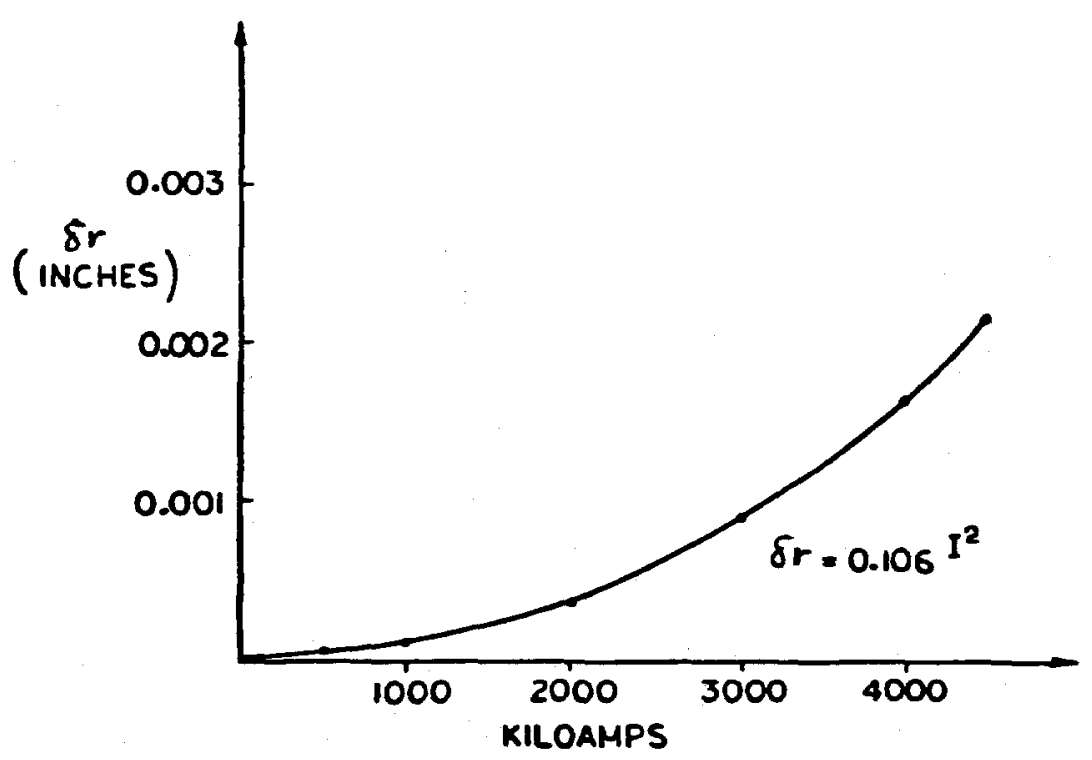

Figure 12. Radial Deflection vs Magnet Current

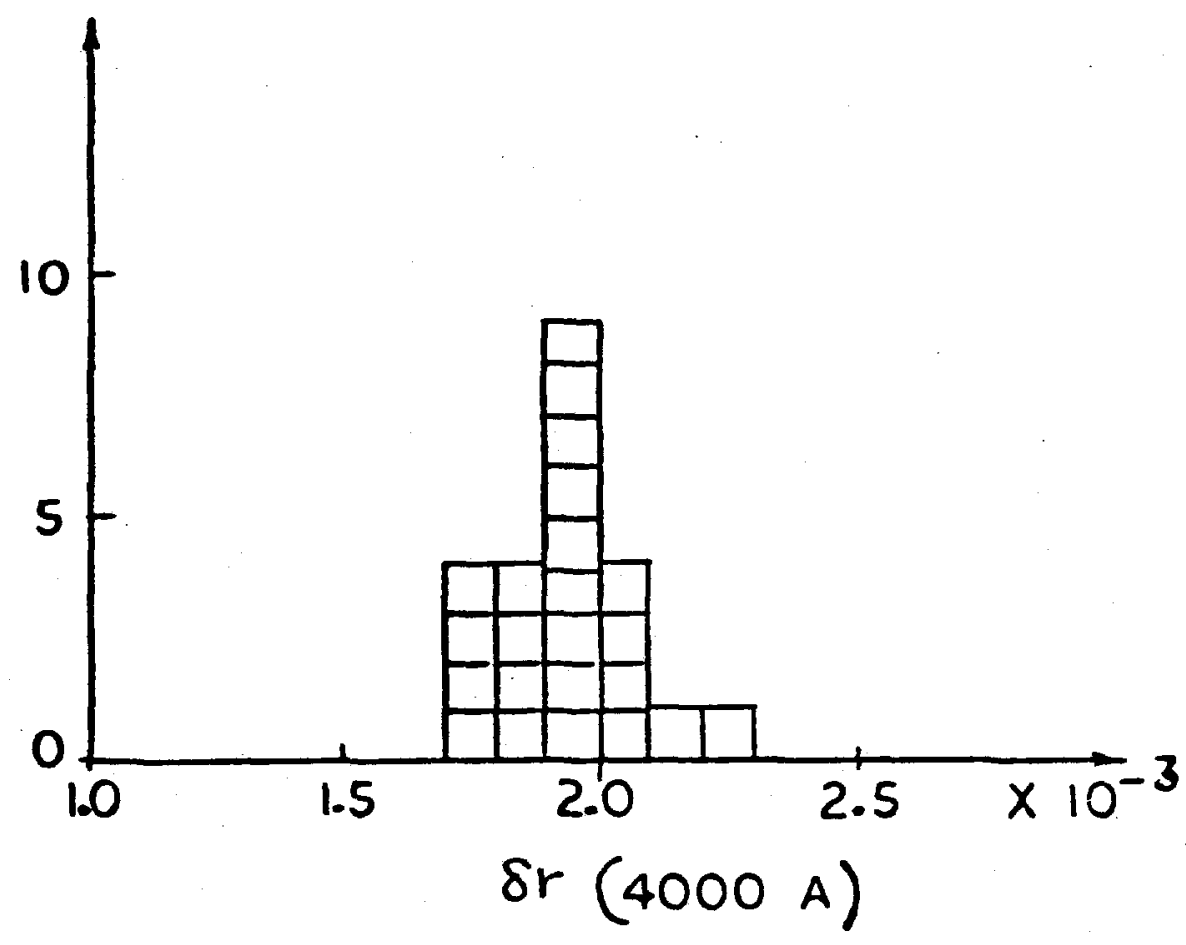

Figure 13. Distribution of $\delta r$ at 4000 Amps 
set of data plus assembly data is stored on a DISC File as permanent data for each magnet. So far about 40 magnets have had a complete set of measurements made, and almost 80 have had partial measurements. We can now examine some of this data.

Figure 14 shows a histogram of the quench current at $200 \mathrm{~A} / \mathrm{sec}$. The effects of different types of eonductors can be seen in Figure 15a. Figure 15b shows a histogram of the magnet effective length. Some identifiable structure changes took place and account for some of the spread in the histogram.

Both the normal and skew harmonics are measured up to 30-pole. The following Table lists the harmonics averaged over 24 magnets.

\section{TABLE I}

Average Limits

\begin{tabular}{|c|c|c|c|c|c|}
\hline K & Pole & $A(k)$ & $B(k)$ & $A(k)$ & $B(k)$ \\
\hline 1 & Quadrupole & $1.78 \pm 3.18$ & $-0.16 \pm 1.65$ & \pm 2.5 & \pm 2.5 \\
\hline 2 & Sextupole & $0.58 \pm 0.89$ & $-9.20 \pm 6.15$ & \pm 1.6 & \pm 6.4 \\
\hline 3 & Octupole & $-0.16 \pm 1.72$ & $0.33 \pm 0.71$ & \pm 4 & \pm 4 \\
\hline 4 & & $0.11 \pm 0.62$ & $3.69 \pm 1.87$ & & \\
\hline 5 & & $-0.18 \pm 0.48$ & $0.33 \pm 0.40$ & & \\
\hline 6 & & $-0.26 \pm 0.38$ & $3.41 \pm 0.49$ & & \\
\hline 7 & & $0.15 \pm 0.34$ & $-0.01 \pm 0.36$ & & \\
\hline 8 & & $0.21 \pm 0.67$ & $-12.96 \pm 0.41$ & & \\
\hline 9 & & $0.36 \pm 0.45$ & $0.06 \pm 0.45$ & & \\
\hline 10 & & $-0.00 \pm 0.45$ & $4.42 \pm 0.37$ & & \\
\hline 11 & & $-0.16 \pm 0.34$ & $0.08 \pm 0.37$ & & \\
\hline 12 & & $0.00 \pm 0.37$ & $-1.13 \pm 0.36$ & & \\
\hline 13 & & $0.06 \pm 0.42$ & $-0.04 \pm 0.53$ & & \\
\hline 14 & 30-pole & $-0.01 \pm 0.40$ & $-0.01 \pm 0.33$ & & \\
\hline & & \multicolumn{2}{|c|}{ All units $\frac{\delta B \cdot \times 10^{4}}{B}$ at $1^{4}$} & & \\
\hline
\end{tabular}




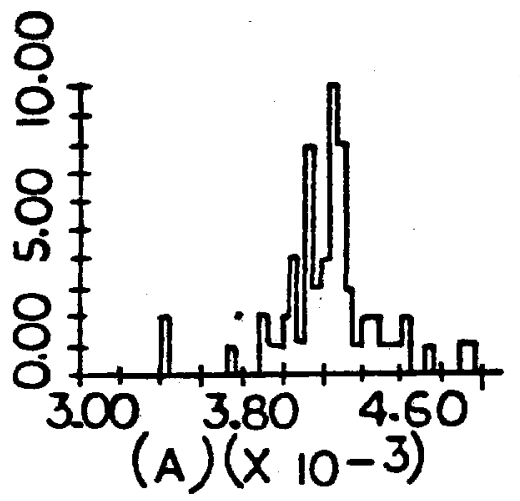

Figure 14. Distribution of $I_{Q}$ at $\dot{I}=200 \mathrm{~A} / \mathrm{sec}$

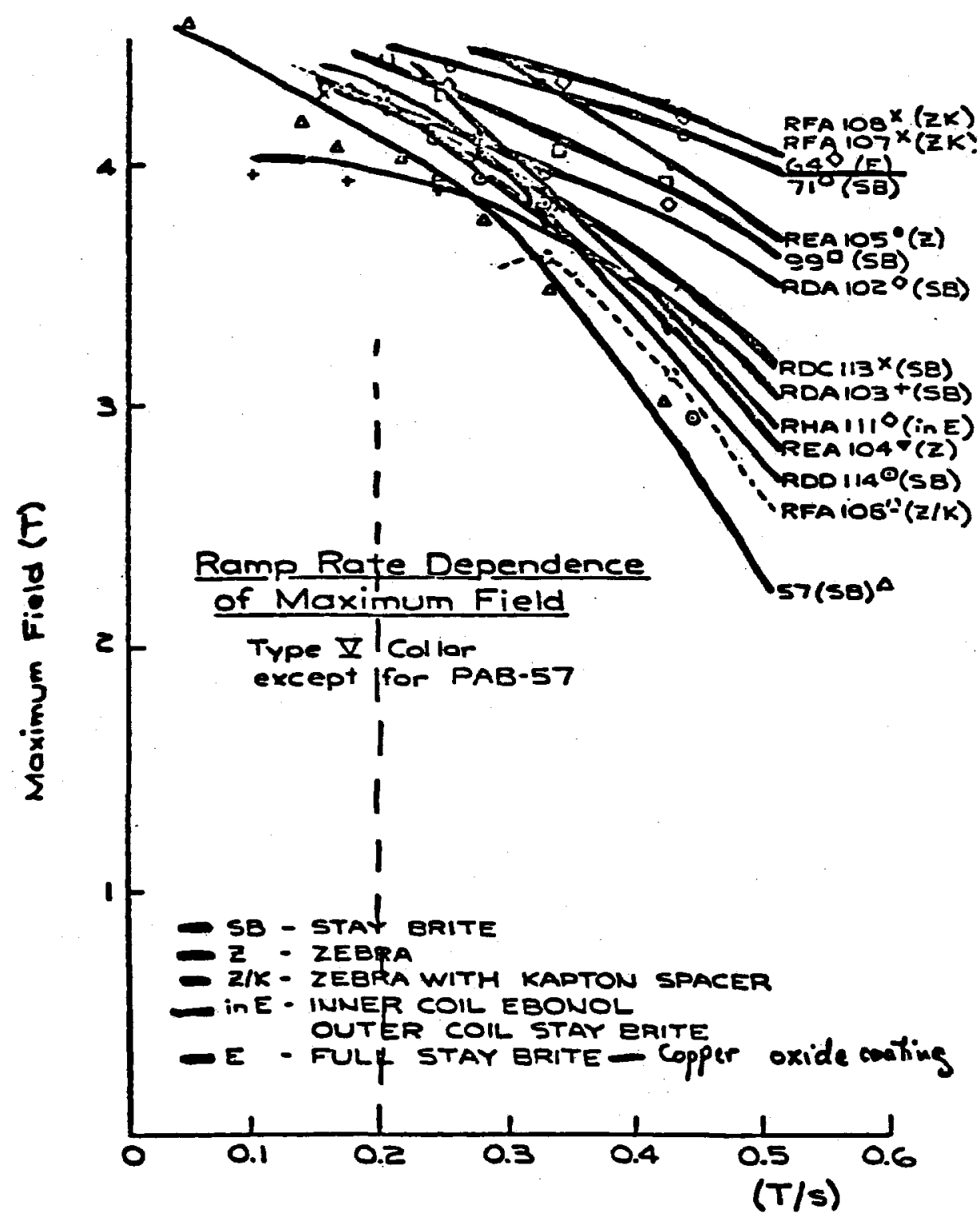

Figure 15a. Ramp Rate Dependence of Magnets in Cryostats 


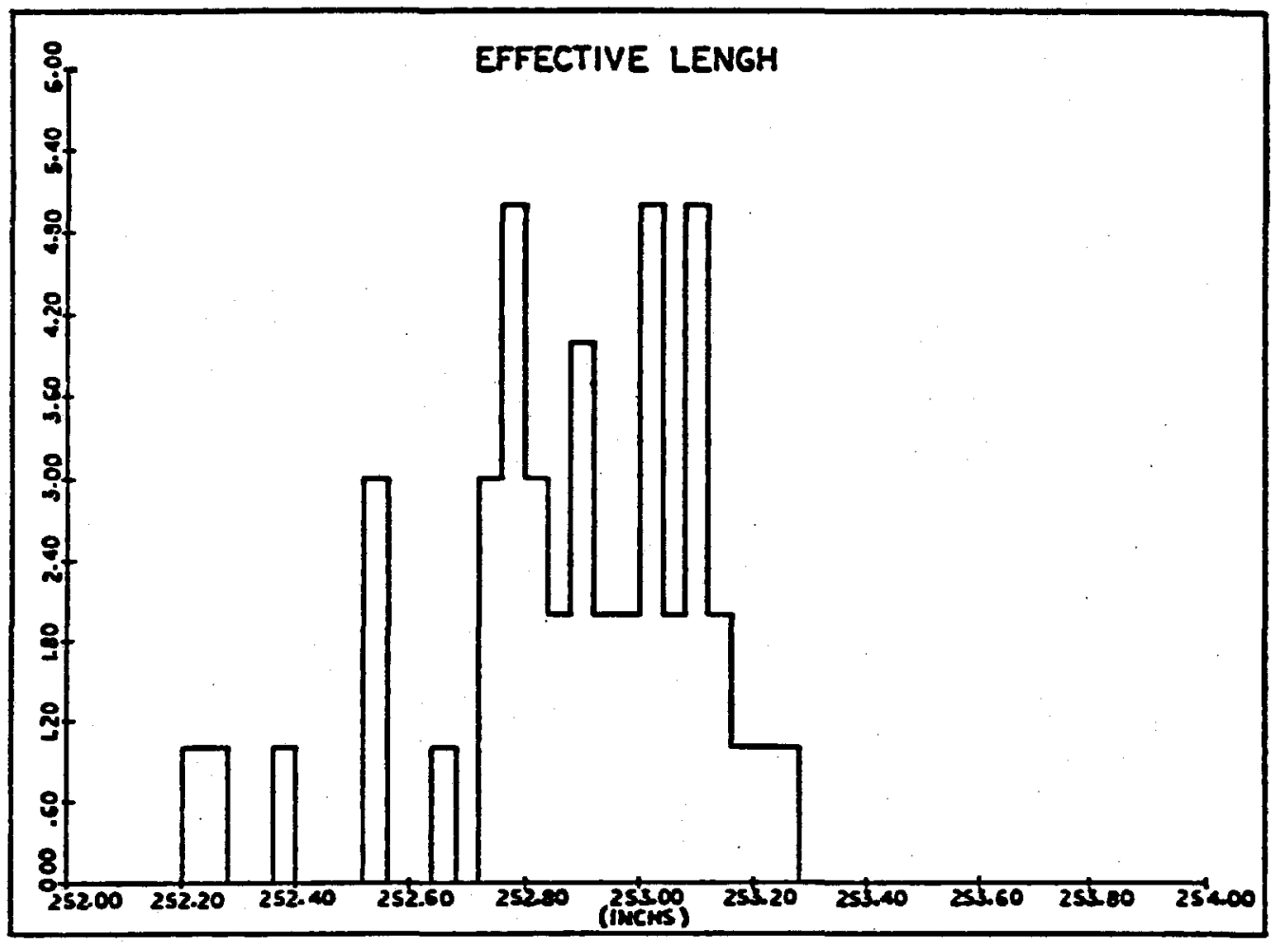

Figure 15b. Effective Length at $2 \mathrm{~T}$

All of the $a_{n}$ and the odd $b_{n}$ should be missing in an ideal magnet. These terms therefore give some indication of the random errors generated during coil fabrication. The even $b_{n}$ are permitted and their values are listed on page 9, along with the design value. It is seen that above the decapole the design values agree well with the desired ones. The sextupole and decapole are not correct. These will be corrected by adjusting the shims at the keys. The line labeled "Limits" indicates the results of theoretical studies on the acceptable limits for various multipoles in the Tevatron. This study is not yet finished and work is still in progress. Limits on multipoles not listed will shortly be specified.

Small effects due to coil motion and persistent currents in the superconductor cause the multipole movements to be current dependent. 
Eddy current effects are also present in the cable strands. All of these effects mainly show up in the sextupole moment. Eddy currents in the Ebonol winding are too small to measure, but Figure 16 shows the hysteresis in the sextupole moment $b_{2}$. Dynamic corrections are planned to cancel out this variation. The results of many other measurements are given in Reference 9.

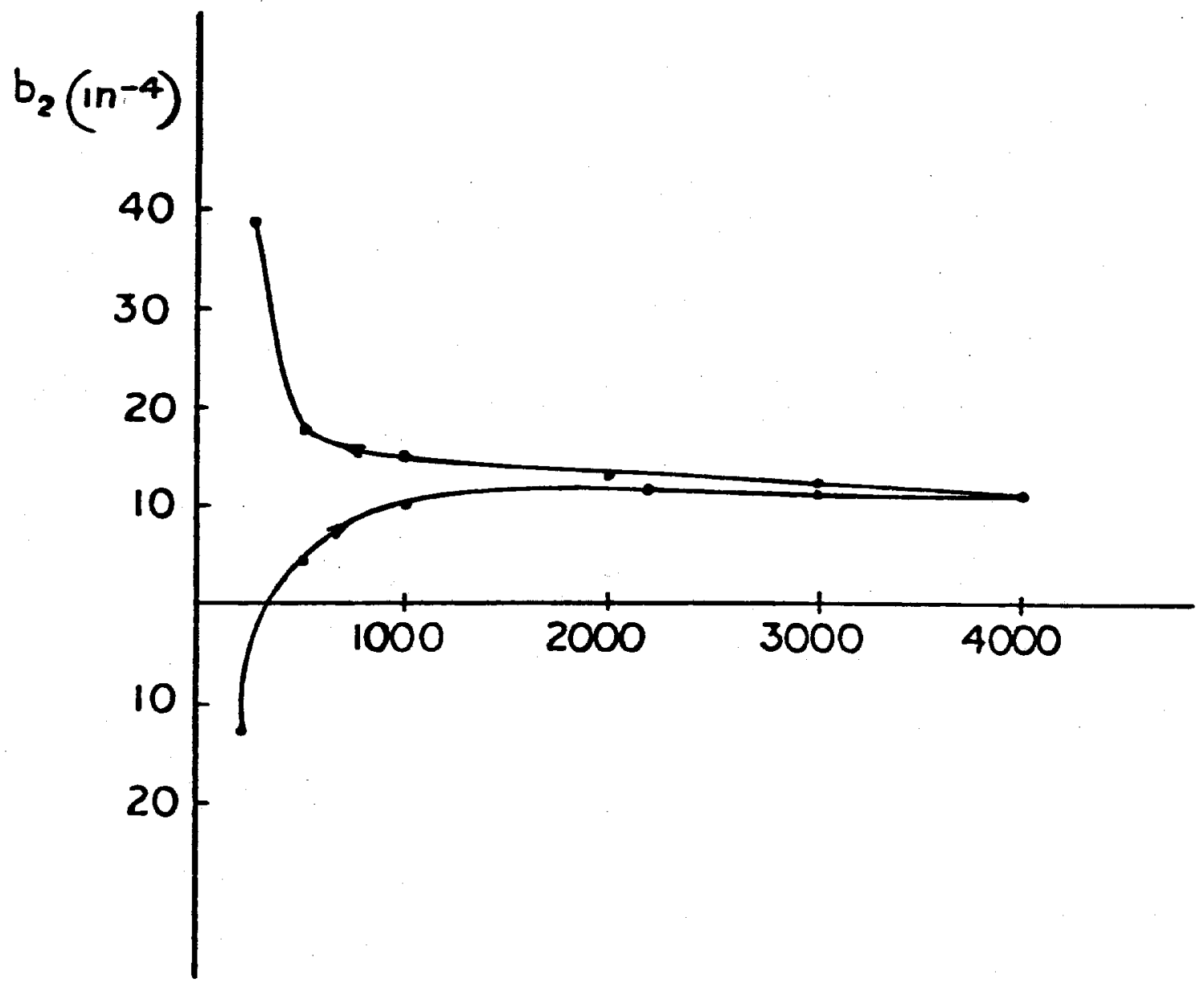

Figure 16. Sextupole Moment vs Magnet Current

BEHAVIOR OF STRING OF MAGNETS

At present tests on strings of magnets are being intensively studied. Three types of tests have been conducted. 
First, strings of four to eight magnets have been cooled, pulsed to full field and their behavior under quench conditions studied. 10 These tests were above ground and no beam was involved and their primary option has been to develop the quench protection system and a prototype power supply. Cryogenic tests have also been made, which verify the initial calculations of the static heat load of a magnet being about 6 watts.

Figure 17 shows the enthalpy of the coil itself, the coil plus collars, and the helium. It is seen that the helium serves as an energy "ballast" at operating temperature and that a full energy quench with all of the energy uniformily deposited throughout only the coil would raise the coil temperature to no more than $100^{\circ} \mathrm{K}$. The main function of the heaters that are fired when a quench is detected is to bring as much of the coil as possible into active energy absorption. The crosses show the temperature actually recorded in the magnet structure gas at about 2-3 minutes after a quench. It is seen that some fraction of the energy is carried off by the helium. This is not yet quantitatively understood and further work is in progress to study these effects.

Figure $18 \mathrm{a}, \mathrm{b}$, shows the $\int \mathrm{I}^{2} \mathrm{dt}$ and the pressure in the cryostat vs the current in the magnets for induced quenches in a string of four magnets.

In the near future the string of four magnets will be increased to eight and then 16 -- which is close to half of a "cryogenic string". In addition to tests above ground, three series of tests have been carried out with beams of protons passing through the magnets. ${ }^{11}$ We reproduce here a curve from the study by Cox which shows the variation of the number of protons at $400 \mathrm{GeV}$ hitting the magnets that will 


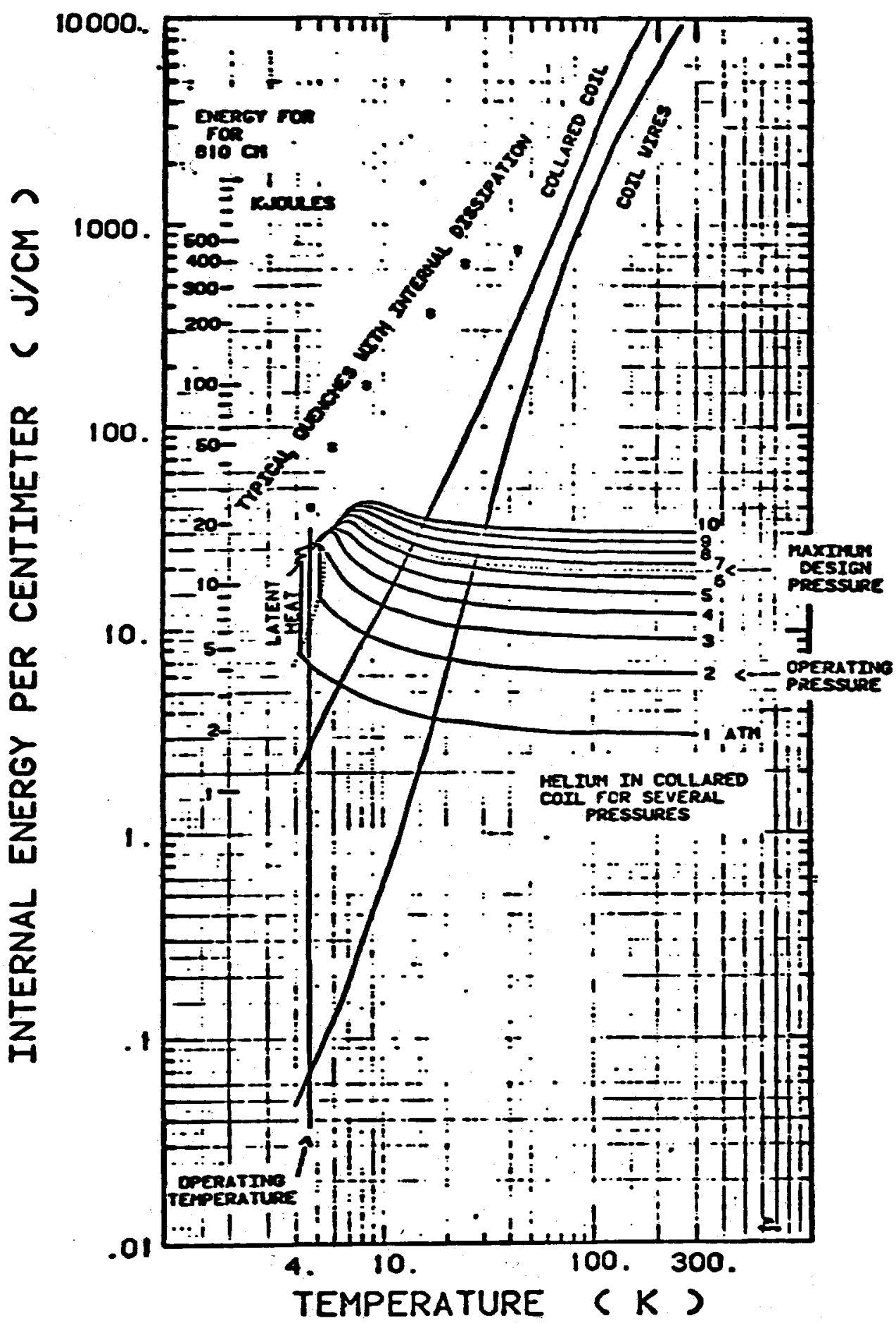

Figure 17. Enthalpy of Coil and Magnet Structures 


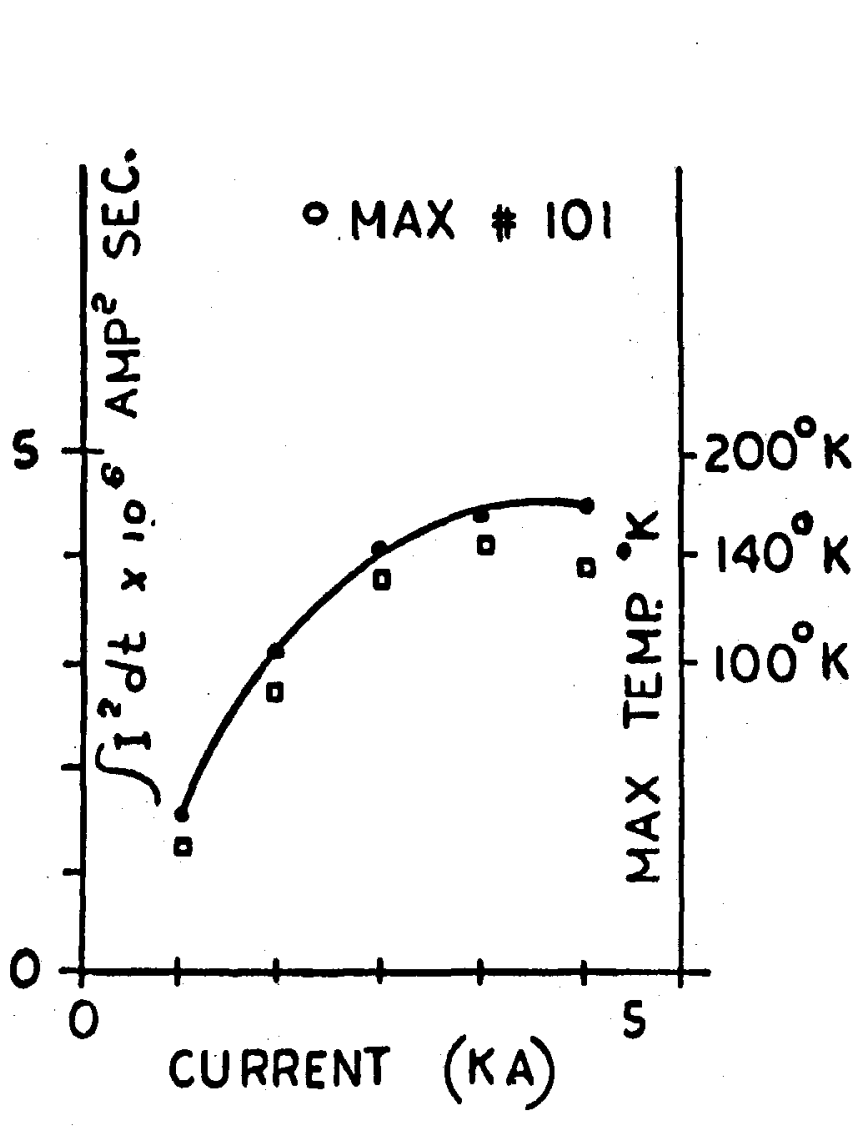

Figure 18a.

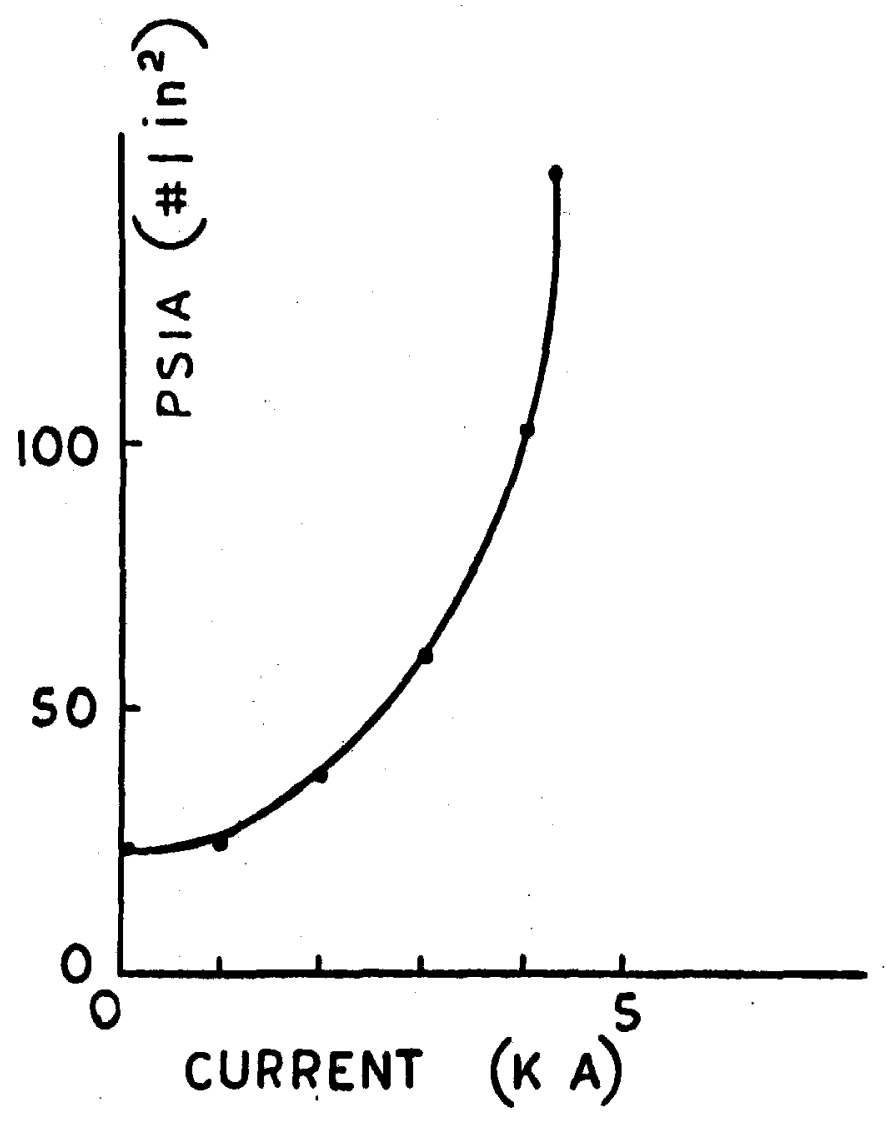

Figure 18b.

$\int I^{2} d t$ and the Pressure In the Cryostat vo the Current in the Magnets 
cause a quench vs the magnet current. The magnet has a no beam quench Iimit of about $4000 \mathrm{~A}$. Calibration of the beam indicated that at $3500 \mathrm{~A}$ in the magnet, $4 \mathrm{~mW} / \mathrm{g}$ for slow extraction, or about $1 \mathrm{~mJ} / \mathrm{g}$ per pulse ( 1 msec) for fast extraction will cause quenching of the magnets. Extensive studies of technique for shielding the magnets during extraction have been carried out, ${ }^{12}$ and techniques are being developed so that the Tevatron can provide both slow as well as fast extracted beam. Finally, a string of 24 magnets and their associated quadrupoles are installed in the Main Ring tunnel and are being cooled by a satellite refrigerator. (See Figure 18.) Beam was first passed through the string at $90 \mathrm{GeV}$ in January 1979. Much experience has been gained in learning how to install and cool Doubler magnets under the actual conditions that must finally be faced. At the low injection energy it was very pleasant to discover that the string of magnets would recover from a beam induced quench in less than $30 \mathrm{sec}$. These studies are being continued, but full field studies will not be carried out until tests above ground on smaller strings of magnets have been studied in greater detail.

\section{SUMMARY}

The program at Fermilab is now about ready to go into production of accelerator-quality magnets suitable for installing in the final Tevatron ring. It is felt that the major problems of producing high quality, rugged, vacuum-tight and cryogenically acceptable magnets have been overcome. It is hoped that a major step toward applying superconductivity to accelerator magnets has been mastered and that the way is opened to applying these techniques to still higher energy machines in the future. 


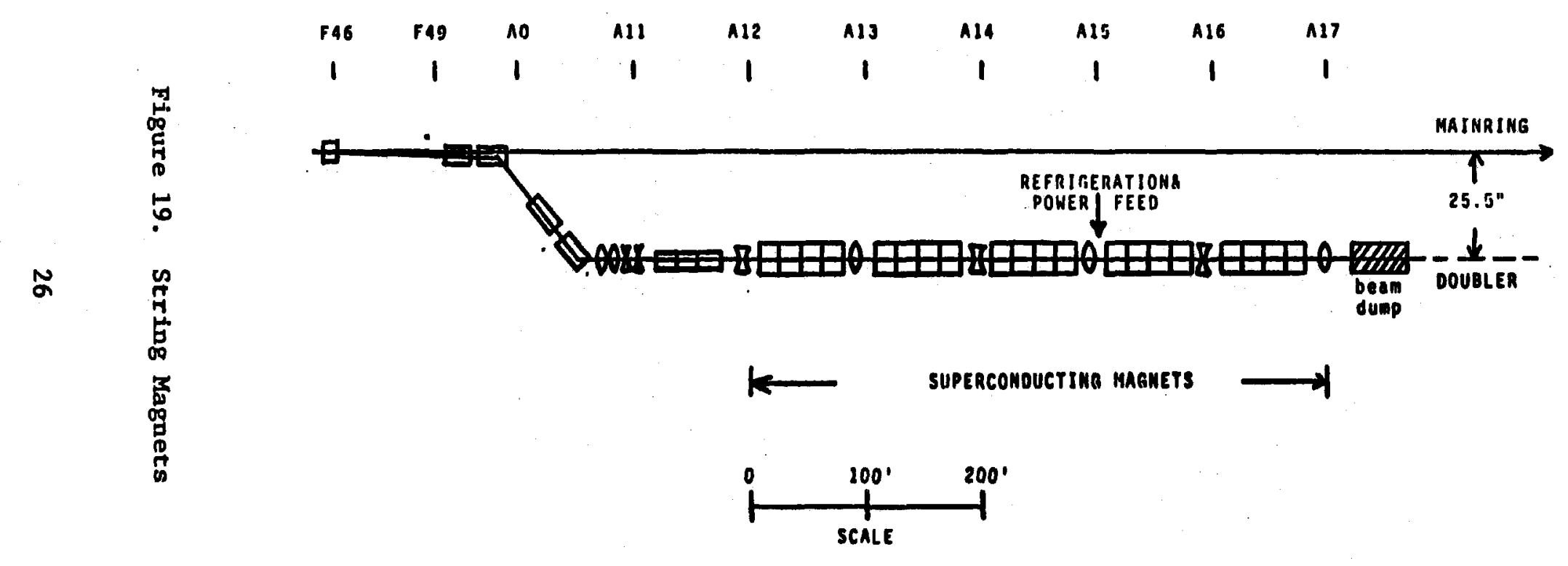




\section{REFERENCES}

1. Project Description:

a. The Energy Doubler - A Progress Report for the Energy Doubler, Saver, Collider Project, Fermilab, June 1976.

b. R.R.Wilson, The Tevatron, Phys. Today, 23-30 (October 1977).

c. The Energy Saver, Fermilab Report, January 1978.

d. H.T.Edwards et al, Report on the Design of the Fermilab Superconducting Accelerator, Fermilab, May 1979.

e. A.V.Tollestrup, Progress Report -- Fermilab Energy Doubler, IEEE Trans. Magn., MAG-15, No. 1, 647 (January 1979).

2. Magnet Power Supply and Quench Protection System:

a. R.Stiening et al, A Superconducting Synchrotron Power Supply and Quench Protection Scheme, IEEE Trans. Magn., MAG-15, No. 1, 670 (January 1979).

b. R.H.Flora and G.S.Tool, Doubler-Tevatron HP Quench Protection System, Paper D-47 presented at the 1979 Particle Accelerator Conference, San Francisco, CA, March 12-14, 1979.

3. Refrigeration System:

a. R. Rihel et al, Central Liquefier for the Fermilab Tevatron, Paper J-32 presented at the 1979 Particle Accelerator Conference, San Francisco, CA, March 12-14, 1979.

b. A.Visser et al, Power for the Fermilab Tevatron Helium Liquefier, Paper K-21 presented at the 1979 Particle Accelerator Conference, San Francisco, CA, March 12-14, 1979.

4. Mechanical Structure of Magnet:

a. G.Biallas et al, The Support and Cryostat System for Doubler Magnets, IEEE Trans. Magn., MAG-15, No. 1, 131 (January 1979). 
5. Fabrication of Magnet Coils:

a. K. Koepke et al, Fermilab Doubler Magnet Design and Fabrication Techniques, IEEE Trans. Magn., MAG-15, No. 1, 658 (January 1979)

6. Room Temperature Field Measurements:

a. R.E.Peters et al, Room Temperature Field Measurements of Superconducting Magnets, IEEE Trans.` Magn., MAG-15, No. 1, 134 (January 1979).

7. Results of Vertical Dewar Tests:

a. A.V.Tollestrup, Progress Report - Fermilab Energy Doubler, IEEE Trans. Magn., MAG-15, No. 1, 647 (January 1979).

8. Cryostat Production:

a. B.P.Strauss et al, How to Mass Produce Reliable Cryostats for Large Particle Accelerators, Paper K-22 presented at the 1979 Particle Accelerator Conference, San Francisco, CA, March 1214, 1979.

9. Magnet Test Facility:

a. D.A.Gross et al, Production Measurement of Energy Doubler Magnets, IEEE Trans. Magn., MAG-15, No. 1, 137 (January 1979 ).

b. M.Wake et al, AC Loss in Energy Doubler Magnets, IEEE Trans. Magn., MAG-15, No. 1, 141 (January 1979).

c. D.A.Gross et al, Results of Recent Measurements of Energy Doubler/Saver Magnets, Paper K-7 presented at the 1979 Particle Accelerator Conference, San Francisco, CA, March 12-14, 1979.

10. Multiple Magnet Test Program:

a. P.Brindza et al, The Multiple Magnet Test Program, Paper K-5 presented at the 1979 Particle Accelerator Conference, San 
Francisco, CA, March 12-14, 1979.

11. Measurements of the Effect of Beam Striking Energy Doubler/Saver Magnets :

a. B.Cox et a1, Sensitivity of an Energy Doubler Dipole to Beam Induced Quenches, Fermilab Technical Memo 828-A, November 1978.

12. Calculations of Effect of Beam Striking Energy Doubler/Saver Magnets :

a. H.T.Edwards et al, Studies on Radiation Shielding of Ener $\equiv y$ Doubler Magnets, Fermilab UPC-30, December 27, 1978, UPC--0 (up-date), January 12, 1979. 